\title{
Design and Synthesis of Lasso-Inspired Peptides with Antibacterial Activity
}

\author{
François Bédard ${ }^{1}$, Riadh Hammami², Ahmed Gomaa², Muriel Subirade², \\ Ismail Fliss ${ }^{2}$, and Eric Biron ${ }^{1}$ \\ ${ }^{I}$ Faculty of Pharmacy, Université Laval and Laboratory of Medicinal Chemistry, CHU de Québec Research \\ Centre, CHUL, Quebec (QC), G1V 4G2, Canada; ${ }^{2}$ STELA Dairy Research Centre, Institute of Nutrition and \\ Functional Foods, Université Laval, Quebec (QC), G1V 0A6, Canada
}

\section{Introduction}

Microcin J25 (MccJ25) is a 21-residue ribosomally synthesized bactericidal peptide produced by Escherichia coli strains with an unusual lariat protoknot structure [1]. MccJ25 exhibits bactericidal activity toward several Gram-negative food-borne pathogens, including Salmonella, Shigella and E. coli [2]. The particular lasso topology of MccJ25 makes the peptide highly resistant to denaturation by high temperatures or proteolysis. These are attractive properties to both pharmaceutical and food industries. MccJ25 structure consists of an 8-residue cycle (lariat ring) formed by a lactam bond between the N-terminal amine and the $\mathrm{Glu}^{8}$ side chain, which is followed by a 13-residue tail that loops back to thread through the ring (Figure 1). The C-terminal tail (residues 9-21) of the peptide is tightly trapped in the lariat ring due to the presence of two aromatic side chains at positions 19 and 20. RNA polymerase appears to be the principal intracellular target of MccJ25 but other mode of actions have also been identified including inhibition of the respiratory chain [3-5]. SAR studies by site-directed mutagenesis revealed that the inhibitory activity of $\mathrm{MccJ} 25$ tolerates a number of residue substitutions [6]. Recent attempts to produce the lasso structure of MccJ25 by chemical synthesis have not yielded successful microbial inhibitors. Nevertheless, two synthetic peptides derived from MccJ25 without lasso folding were found to be bactericidal [7]. The current lack of information on MccJ25 structure and its essential features for antimicrobial activity could be overcome by chemical engineering and computational studies. We hypothesized that lasso formation is important but not a prerequisite for the activity of MccJ25 and that it may be possible to obtain derivatives that are active without the lasso structure [8]. In this study, we report synthetic peptides based on the MccJ25 sequence but devoid of lasso folding yet retaining activity against bacteria (S. enterica and E. coli) and specific intracellular targets (RNA polymerase and the respiration chain).

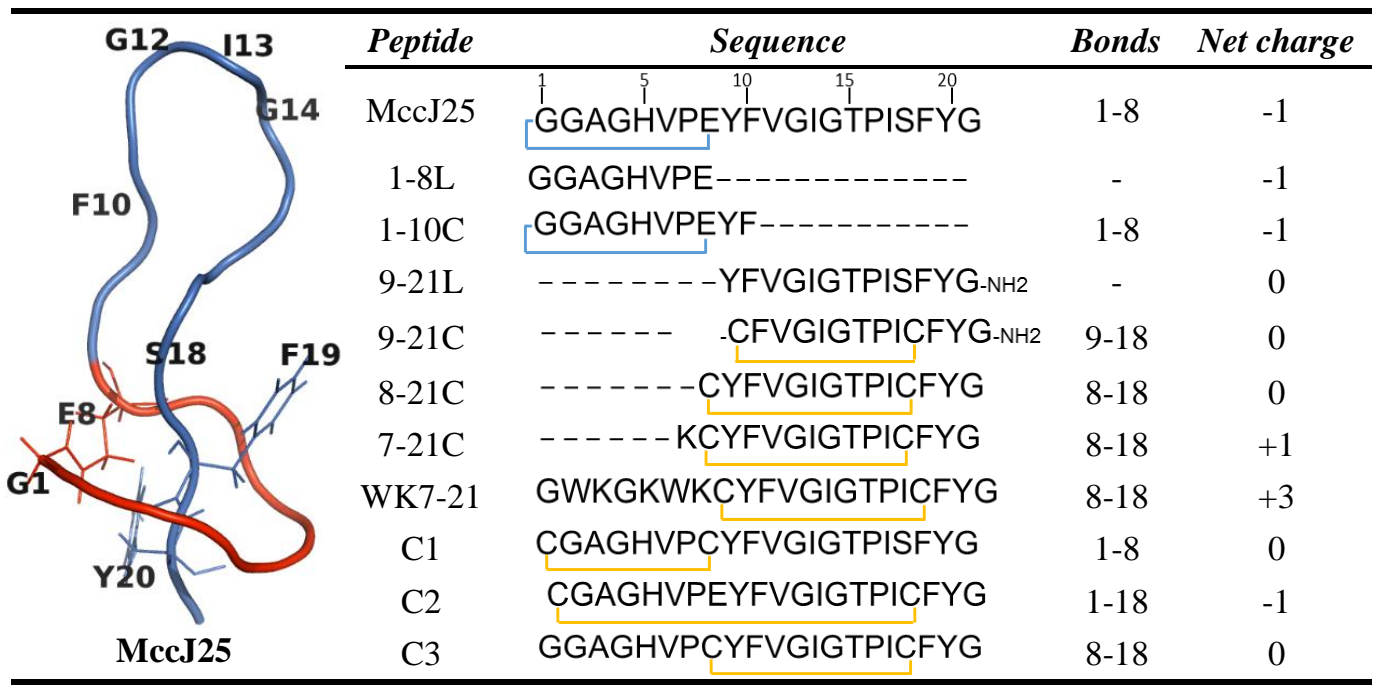

Fig. 1. Amino acid sequence of MccJ25 and derived-peptides used in this study. Amide and disulfide bonds are colored in blue and yellow respectively. 
Table 1. Minimal inhibitory concentrations of MccJ25 and its derivatives for bacteria.

\begin{tabular}{lccccc}
\hline \multirow{2}{*}{ Bacterial strain } & \multicolumn{5}{c}{ Minimal inhibitory concentration $(\mu M)$} \\
\cline { 2 - 6 } & MccJ25* & C1 & $8-21$ C & $7-21$ C & WK7-21 \\
\hline Salmonella enterica ATCC 14028 & 6.5 & $125-250$ & - & - & - \\
Salmonella enterica ATCC 8387 & 0.1 & 1.0 & 1.0 & $7.8-15.6$ & 7.8 \\
Salmonella enterica ATCC 29628 & 6.5 & - & - & - & - \\
Salmonella enterica ATCC 8400 & 0.8 & $62.5-125$ & - & $125-250$ & 62.5 \\
Salmonella enterica ATCC 9607 & 1.6 & 250 & - & - & - \\
Salmonella enterica ATCC 9700 & 0.4 & 250 & - & - & - \\
Escherichia coli ATCC 11229 & 0.2 & 62.5 & $125-250$ & - & 250 \\
Escherichia coli ATCC 25922 & 3.3 & 250.0 & - & 250 & - \\
Escherichia coli ATCC 15144 & - & - & - & - & - \\
Escherichia coli O157:H7 ATCC 35150 & - & - & - & - & - \\
Escherichia coli MC4100 ATCC 35695 & 6.5 & $31.3-62.5$ & - & 125 & - \\
Escherichia coli DH5a & 6.5 & - & - & - & - \\
Listeria ivanovii HPB28 & - & $>250$ & - & $>250$ & 250 \\
Staphylococcus aureus ATCC 6538 & - & $>250$ & - & ND & $>250$ \\
\hline
\end{tabular}

"Produced by bacteria; “-” = no activity detected; $N D$ = not determined

\section{Results and Discussion}

In this study, a series of peptides based on the primary structure of MccJ25 and lacking the lasso structure was first designed in silico, prepared by standard Fmoc solid-phase peptide synthesis (Figure 1) and then evaluated for antibacterial activity [8]. The peptide code is based on MccJ25 numbering. Shortened $N$-terminal and $C$-terminal sequences were included in the design since cleaved MccJ25 has been shown previously to be antibacterial [9]. The lariat ring was synthesized in linear 1-8L and cyclized 1-10C forms. The tail ( $C$-terminal) portion was synthesized as linear peptide 9-21L. A disulfide bond was introduced between $\mathrm{C} 9$ and $\mathrm{C} 18$ to form the head-to-tail circular peptide 9-21C. Peptides 8-21C, 7-21C and WK7-21 were designed from a tail that was scaffold-stabilized using a disulfide bond between Cys residues at positions 8 and 18. While a single Lys residue was inserted at the $N$-terminus of 7-21C, peptide WK7-21 was obtained by substituting the $N$-terminal portion of MccJ25 with multiple hydrophobic (Trp) and basic (Lys) and residues $\left(\mathrm{H}_{2} \mathrm{~N}-\mathrm{GWKGKWK}\right)$ to increase solubility and possibly induce $\beta$-hairpin structure. In addition, peptides $\mathbf{C 1}, \mathbf{C 2}$ and $\mathbf{C 3}$ containing a single disulfide bond (1-8, 1-18 or 8-18) were designed to retain the core structure of the native MccJ25 peptide regardless of the lariat protoknot structure.

Table 1 summarizes the minimum inhibitory activities (MICs) of the synthesized peptides that had activity above $250 \mu \mathrm{M}$ in comparison to bacteria produced microcin. The MccJ25 peptide was active at $\mu \mathrm{M}$ and $\mathrm{nM}$ concentrations (0.1-6.5) against Gram-negative bacteria, with $S$. enterica ATCC 8387 being the most sensitive strain $(\mathrm{MIC}=0.1 \mu \mathrm{M})$. While peptide $\mathbf{C 2}$ was not inhibitory, $\mathbf{C 1}$ inhibited several strains of $S$. enterica and $E$. coli. Against $S$. enterica ATCC 8387, the MICs of peptides C1, C3, 8-21C, 7-21C and WK7-21 were respectively 1.0 $\mu \mathrm{M}, 15.6-31.3 \mu \mathrm{M}, 1.0 \mu \mathrm{M}, 7.8-15.6 \mu \mathrm{M}$ and $7.8 \mu \mathrm{M}$. Inhibition of $S$. enterica ATCC 8387 by MccJ25 and its derived peptides at 0.5 and $7.8 \mu \mathrm{M}$ is summarized in Figure 2A. At $0.5 \mu \mathrm{M}$, the derived peptides were moderately inhibitory (25-50\%). Similarly to MccJ25, inhibition by C1, 8-21C and WK7-21 was total at 7.8 $\mu$ M (above the MIC). Although MccJ25 was not active against Gram-positive bacteria, peptides C1, 7-21C and WK7-21 were weakly inhibitory to Listeria ivanovii HPB28, Staphylococcus aureus ATCC 6538 and Enterococcus faecalis ATCC 27275 (MIC $\geq 250 \mu \mathrm{M}$ ). Peptides MccJ25, C1, 7-21C and WK7-21 did 
not show hemolytic activity against horse erythrocytes at concentrations up to $(50 \mu \mathrm{M})$, which is consistent with the low toxicity reported in the literature [10].

Of the synthetic MccJ25-derived peptides described previously, two in particular, namely GGACHVPEYFVGIGTPISFC (P1, bonded 1-8, 4-20) and CGAGFHVPCYFVGRGTPISFYG (P6, bonded 1-9), were inhibitory to Salmonella newport at MICs of 25 and $30 \mu \mathrm{M}$ respectively [7]. While peptide $\mathbf{C 1}$ of this study was designed with the replacement of the amide bond between G1 and E8 by a disulfide bond, P6 contained besides a Phe insertion at position 5 and an Arg substitution for Ile at position 13. Although these mutations increased the solubility of P6, they decreased its inhibitory action. While peptide $\mathbf{C 1}$ was inhibitory to Gram-negative pathogens, $\mathbf{P 6}$ was 60 times less potent than MccJ25 against $S$. newport and $\mathbf{C 1}$ against $S$. enterica ATCC 8387 was 10 times less potent than MccJ25. Further, $\mathbf{C 1}$ showed a weak activity against Gram-positive bacteria (MIC $>250 \mu \mathrm{M})$. Soudy, et al. [7] also reported absence of activity for a $\mathbf{P 6}$ variant with an 8-residue lariat ring. Based on our results, the Gly substitution by Phe at position 4 could explain the decrease of activity observed for P6 and its variant compared to $\mathbf{C 1}$. The Arg substitution at position 13 has been reported previously to increase the antimicrobial activity of MccJ25 [6]. In this study, different synthetic derivatives of MccJ25 containing substitutions and/or truncations exhibited antibacterial activity against Gramnegative bacteria. Although the reduced activity of these designed synthetic derivatives compared to the native MccJ25, the lasso fold does not seem to be a prerequisite for the antimicrobial activity.

The inhibition of RNAP by MccJ25, 9-21L, C1, 7-21C and WK7-21 was examined in vitro (Figure 2B). Rifampicin, an antibiotic that targets bacterial RNAP was used as a control. MccJ25 was the best inhibitor, reducing $E$. coli RNAP activity by $86.3 \%$ and $97.9 \%$ respectively at 5 and $50 \mu \mathrm{M}$. Peptides C1, 7-21C or WK7-21 at a concentration of $5 \mu \mathrm{M}$ reduced activity by $23.4 \%, 37.4 \%$ and $65.0 \%$ respectively. Linear peptide 9-21L was not inhibitory. At $50 \mu \mathrm{M}$, reductions by MccJ25, C1 and WK7-21 were respectively 97.9\%, 95.7\% and 94.7\%. These results suggest that the MccJ25derived peptides could inhibit $E$. coli by interacting directly with RNAP and thereby interfering with transcription. The ability of MccJ25, C1, 7-21C and WK7-21 to inhibit respiration of $S$. enterica ATTC 8387 is shown in terms of MIC in Figure 2C. The decrease in oxygen consumption by $S$. enterica after incubation with MccJ25 was $57.2 \%$. WK7-21 was the strongest inhibitor among the synthetic peptides, decreasing oxygen consumption by $36.9 \%$, followed by $\mathbf{C 1}$ and $\mathbf{7 - 2 1 C}$ at $32.1 \%$ and $24.2 \%$, respectively. Inhibition of respiration by peptides WK7-21, C1 and 7-21C suggests that they share at least one mechanism of action with MccJ25. In this study, a series of MccJ25-derived peptides lacking the lasso structure was designed by an in silico approach. Some of the designed peptides were inhibitory to $S$. enterica and E. coli. Since C1, 7-21C and WK7-21 were all at least ten
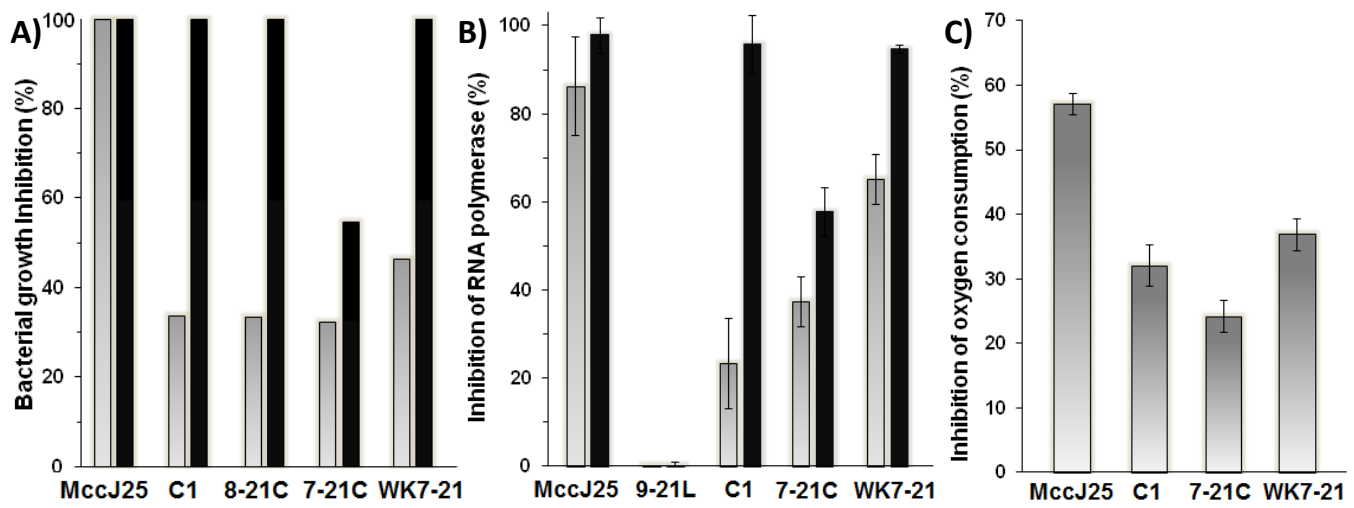

Fig. 2. A) Bacterial growth inhibition of MccJ25, C1, 8-21C, 7-21C and WK7-21 at $0.5 \mu M$ (gray) and $7.8 \mu \mathrm{M}$ (black) against Salmonella enterica subsp. enterica ATCC 8387. Data are means of triplicate measurements. B) Inhibition of E. coli RNA polymerase in vitro by MccJ25, 9-21L, C1, 7-21C and WK7-21 at $5 \mu \mathrm{M}$ (gray) and $50 \mu \mathrm{M}$ (black). Rifampicin (200 nM) was the positive control. The values are means $\pm S D$ of triplicate analyses. $C$ ) Inhibition of oxygen consumption by $M c c J 25, C 1,7-21 C$ and $\mathbf{W K} 7-21$ at their respective MICs. The values are means $\pm S D$ of triplicate analyses. 
times less potent than MccJ25, the constrained lasso structure might be more than somewhat important for antibacterial action. MccJ25 inhibits transcription by binding in the secondary channel of RNAP and thereby blocking substrate access to the catalytic site. The Tyr ${ }^{9}$ residue of MccJ25 has been shown essential for RNAP inhibition with no compatible substitutions [6]. Other residues, namely $\mathrm{Gly}^{4}$, Pro $^{7}$, $\mathrm{Phe}^{10}$ and Phe ${ }^{19}$ are reportedly important but not strictly essential for MccJ25 binding to RNAP [6]. In the present study, we observed that MccJ25-derived peptides lacking the lasso structure were able to inhibit in vitro RNAP at high concentrations $(5$ and $50 \mu \mathrm{M})$. Although these peptides presumably bound to RNAP, it is not possible to deduce that they share a common binding site with MccJ25. Pavlova, et al. [6] reported that the RNAP/MccJ25 interaction involves primarily hydrophobic interaction. Since the active MccJ25-derived peptides had Phe residues at positions 10 and 19 and Tyr at position 9, these might be involved in the inhibition of RNAP. On the other hand, in the case of WK 7-21, the KWK pattern at the $N$-terminus could be involved. This pattern is known to bind to DNA [11]. The respiratory apparatus of $S$. enterica could be a target for WK7-21, C1 and 7-21C, as it is for MccJ25. Uptake of these peptides inside cells leads to increased superoxide production, oxidative damage of biologically important molecules, and ultimately cell death. Further studies are required in order to decipher the precise molecular mechanism of these and other MccJ25-derived peptides.

These findings put forward the possibility of producing MccJ25-derived peptides lacking the lasso structure but nevertheless conserving antibacterial activity. The presence of the lasso constrains the structure of MccJ25 while conferring potent antibacterial activity to the peptide. MccJ25-derived peptides lacking the lasso but otherwise constrained in a rigid overall structure, for example by disulfide bonds, could be strongly antibacterial. A rigid topology close to that of $\mathrm{MccJ} 25$ appears to make the molecule a more potent antibacterial agent.

\section{Acknowledgments}

François Bédard thanks the Fonds de recherche du Québec - Nature et technologies (FQRNT) and the National Sciences and Engineering Research Council of Canada (NSERC) for graduate and undergraduate scholarships, respectively. This work was supported by the FRQNT (2011-PR-139387).

\section{References}

1. Bayro, M.J., et al. J. Am. Chem. Soc. 125, 12382-12383 (2003), http://dx.doi.org/10.1021/ja036677e

2. Sable, S., Pons, A.M., Gendron-Gaillard, S., Cottenceau, G. Appl. Environ. Microbiol. 66, $4595-4597$ (2000), http://dx.doi.org/10.1128/AEM.66.10.4595-4597.2000

3. Mukhopadhyay, J., Sineva, E., Knight, J., Levy, R.M., Ebright, R.H. Mol. Cell 14, 739-751 (2004),

http://dx.doi.org/10.1016/j.molcel.2004.06.010

4. Bellomio, A., Vincent, P.A., de Arcuri, B.F., Farías, R.N., Morero, R.D. J. Bacteriol. 189, 4180-4186 (2007), http://dx.doi.org/10.1128/jb.00206-07

5. Niklison Chirou, M., Bellomio, A., Dupuy, F., Arcuri, B., Minahk, C., Morero, R. FEBS J. 275, $4088-4096$ (2008), http://dx.doi.org/10.1111/j.1742-4658.2008.06550.x

6. Pavlova, O., Mukhopadhyay, J., Sineva, E., Ebright, R.H., Severinov, K. J. Biol. Chem. 283, 25589-25595 (2008), http://dx.doi.org/10.1074/jbc.M803995200

7. Soudy, R., Wang, L., Kaur, K. Bioorg. Med. Chem. 20, 1794-1800 (2012), http://dx.doi.org/10.1016/j.bmc.2011.12.061

8. Hammami, R., Bédard, F., Gomaa, A., Subirade, M., Biron, E., Fliss, I. Amino Acids, 47, 417-428 (2015), http://dx.doi.org/10.1007/s00726-014-1877-x

9. Blond, A., Cheminant, M., Destoumieux-Garzón, D., Ségalas-Milazzo, I., Peduzzi, J., Goulard, C., Rebuffat, S. Eur. J. Biochem. 269, 6212-6222 (2002), http://dx.doi.org/10.1046/j.1432-1033.2002.03340.x

10. Lopez, F.E., Vincent, P.A., Zenoff, A.M., Salomón, R.A., Farías, R.N. J. Antimicrob. Chemother. 59, 676680 (2007), http://dx.doi.org/10.1093/jac/dkm009

11. Johnson, N.P., Mazarguil, H., Lopez, A. J. Biol. Chem. 271, 19675-19679 (1996), http://dx.doi.org/10.1074/jbc.271.33.19675 